\title{
Angioplastia com Implante de Stents para o Tratamento de Dissecção Coronária Espontânea
}

\author{
Edmílson Y. Ishii ${ }^{1}$, Daniel Chamié1, Rodolfo Staicoํㅜ, Fabrício R. Las Casas¹, Fausto Feres ${ }^{1}$, \\ Alexandre Abizaid', Luiz Alberto P. Mattos' ${ }^{1}$, Áurea Chaves ${ }^{1}$, Amanda G. M. R. Sousa' ${ }^{1}$, J. Eduardo Sousa ${ }^{1}$
}

\section{RESUMO}

É relatado o caso de uma mulher de 40 anos, hipertensa e tabagista, que foi admitida com quadro de infarto agudo do miocárdio sem supradesnivelamento do segmento ST. A cinecoronariografia inicial revelava lesão moderada no terço proximal da artéria circunflexa. Proposto tratamento percutâneo da referida lesão e, no momento do procedimento, a angiografia revelou ausência de lesões na circunflexa e aparecimento de extensa dissecção desde o óstio até o terço médio da artéria descendente anterior, com fluxo distal TIMI III, optandose por tratamento clínico. Durante a internação, a paciente evoluiu com angina recorrente e elevação do segmento ST no eletrocardiograma. Nova cinecoronariografia mostrava extensão da dissecção até o terço distal do vaso, com lentificação do fluxo (TIMI II). Foi então, submetida a angioplastia percutânea com implante de três stents guiado por ultrasom intracoronário, com sucesso.

DESCRITORES: Angioplastia transluminal percutânea coronária. Contenedores. Coronariopatia. Aneurisma dissecante.

D issecção espontânea de artérias coronárias (DCE) é uma causa particularmente rara de síndrome coronariana aguda e morte súbita, com apresentação indistinguível dos quadros decorrentes de ruptura de uma placa aterosclerótica coronariana e, geralmente, relacionadas a mau prognóstico ${ }^{1-5}$.

A maioria dos casos ocorre em mulheres jovens, sem fatores de risco para doença arterial coronariana (DAC), durante o período periparto ou em associação com o uso de contraceptivos orais ${ }^{1-7}$.

A apresentação clínica pode ser angina estável, angina instável, infarto agudo do miocárdio (IAM) ou, mais comumente, morte súbita cardíaca ${ }^{3,7}$.

\footnotetext{
Instituto Dante Pazzanese de Cardiologia.

Correspondência: Edmilson Y. Ishii. Av. Dr. Dante Pazzanese, 500

- Ibirapuera - São Paulo - SP - Brasil - Tel.: (55-11) 5085-4141

Recebido em: 15/01/2007 - Aceito em: 27/02/2007
}

\section{SUMMARY}

Angioplasty with Stent Implantation for the Treatment of Spontaneous Coronary Artery Dissection

This is the description of a 40-year-old hypertensive female patient who was also a smoker. The patient was admitted with non-ST elevation myocardial infarction. Baseline coronary angiography revealed a moderate lesion in the mid third of the circumflex artery. Percutaneous treatment of the lesion was indicated. At angiography no lesion on the circumflex artery could be visible, but instead, an extensive dissection in the left descending coronary artery from the ostium to mid third with TIMI III distal flow. Clinical treatment was the choice. However, while in hospital patient's ECG presented recurrent angina and ST elevation. A new coronary angiography revealed dissection extension to the distal part of the vessel, and slower flow (TIMI II). The patient was then successfully submitted to an intra-coronary ultrasoundguided coronary angioplasty with implant of three stents.

DESCRIPTORS: Angioplasty, transluminal, percutaneous coronary. Stents. Coronary disease. Aneurysm, dissecting.

Na maioria dos casos, o diagnóstico é feito postmortem, com documentação angiográfica limitada ${ }^{1,2,5,7,8}$.

A melhor forma de tratamento permanece indefinida, sendo que as abordagens medicamentosa, percutânea ou cirúrgica devem ser individualizadas para cada caso.

\section{RELATO DO CASO}

Paciente do sexo feminino, 40 anos, com antecedentes de hipertensão arterial (HAS), tabagismo e história familiar de DAC, foi admitida com quadro de dor torácica em aperto, de forte intensidade, com irradiação para o membro superior esquerdo e iniciada em repouso. O eletrocardiograma (ECG) não apresentava alterações significativas, mas a elevação de troponina-I configurou quadro de IAM sem supradesnivelamento de ST (IAM SSST). Foram adotadas medidas clínicas para estabilização do quadro, com aspirina, clopidogrel, heparina de baixo peso molecular, beta-bloqueadores 
e nitrato intravenoso, com melhora do quadro anginoso. Submetida a um estudo cineangiocoronariográfico dois dias após a admissão, que evidenciou uma lesão estenótica moderada em terço proximal da artéria coronária circunflexa $(C x)$ - Figura 1A. As artérias descendente anterior (DA) e coronária direita (CD) eram normais. A função ventricular esquerda estava preservada. Foi, então, programado o tratamento percutâneo da referida lesão. Durante a angiografia inicial para o procedimento, observou-se ausência de lesão na Cx, comprovando que a imagem observada no primeiro exame tratava-se espasmo coronário (Figura 1B). Notou-se, também, presença de extensa dissecção na DA, desde sua origem até o seu terço médio, com fluxo distal TIMI III, ausente na coronariografia inicial (Figura 2). A paciente encontrava-se assintomática e hemodinamicamente estável, optando-se por tratamento clínico.

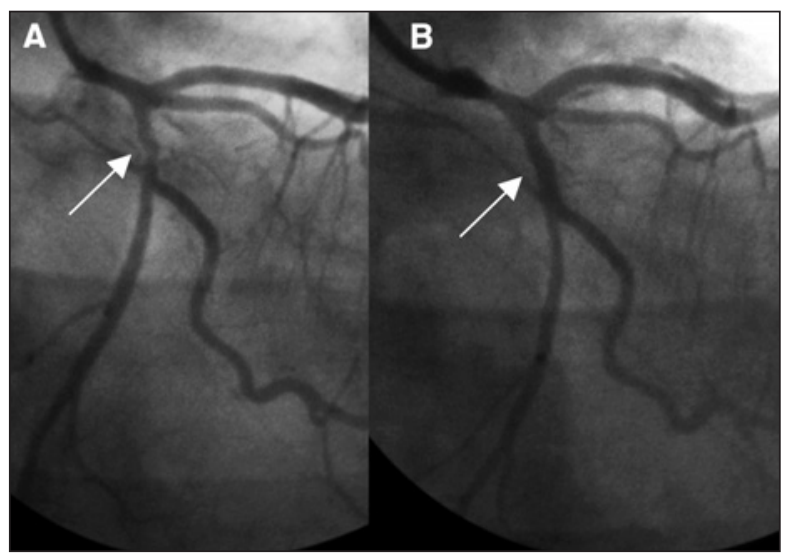

Figura 1 - A) Coronariografia inicial demonstrando lesão obstrutiva moderada no terço proximal da Cx. B) Coronariografia dois dias após revelando ausência de obstrução significativa na $C x$, após estabilização do quadro com medicações anti-isquêmicas, comprovando a presença de espasmo coronário prévio.

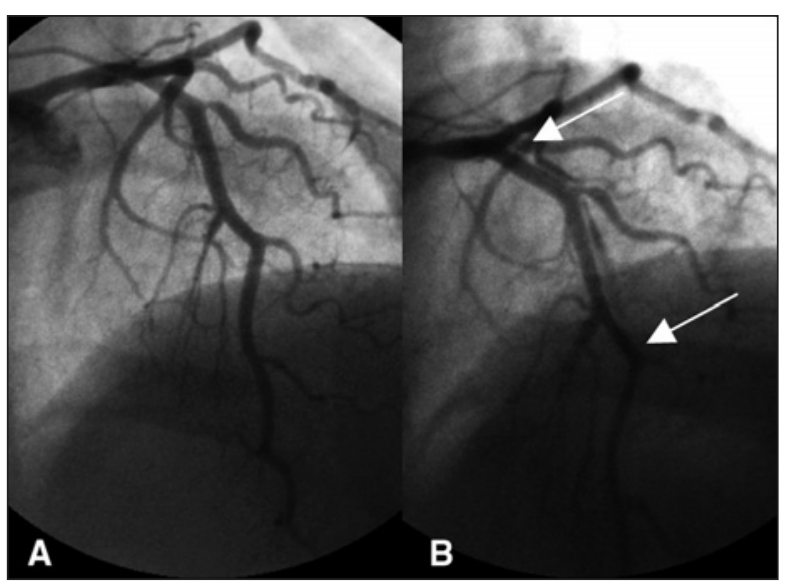

Figura 2 - A) Coronariografia inicial demonstrando aspecto angiográfico normal da DA. B) Segunda coronariografia revelando extensa dissecção na DA, desde seu óstio até seu terço médio, com fluxo TIMI III.
Contudo, 12 dias após, apresentou novo quadro de dor precordial em aperto, de forte intensidade, com sudorese fria e irradiação para o membro superior esquerdo. O ECG, durante o quadro de dor, mostrava supradesnivelamento do segmento ST em parede anterior. Realizada nova cineangiocoronariografia, que demonstrou piora da dissecção na DA, estendendo-se, agora, desde seu óstio até o terço distal, com fluxo TIMI II (Figura 3). A ventriculografia esquerda mostrava hipocinesia $2+/ 4+$ na parede anterior. Tendo em vista a evolução clínica desfavorável e piora do aspecto da dissecção, com comprometimento do fluxo, optamos pelo tratamento percutâneo com implante de stents. O procedimento foi guiado por ultra-som intracoronário (USIC), sendo necessário o implante de três stents longos (um Bx Sonic ${ }^{\circledR} 3,5 \times 33 \mathrm{~mm}$ e, dois Driver $^{\circledR} 3,0 \times 30 \mathrm{~mm}$ ), com sobreposição de hastes. Após o implante, notouse ao USIC aposição incompleta das hastes do stent proximal, tendo sido feita nova pós-dilatação com cateterbalão Maverick ${ }^{\circledR} 4,0 \times 20$. Obteve-se excelente resultado angiográfico final com resolução da dissecção coronária e normalização do fluxo (Figura 4). Houve resolução da dor e do supra do segmento ST no ECG após o procedimento, com a paciente permanecendo assintomática e com boa evolução no período pós-IAM. Recebeu alta hospitalar quatro dias após, em uso de

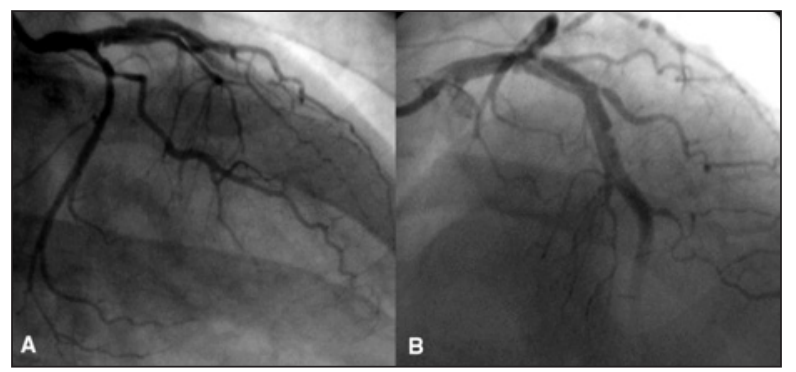

Figura 3 - Coronariografia após instabilização clínica e supradesnível do segmento ST na parede anterior, evidenciando agravamento da dissecção na DA, com comprometimento do seu fluxo (TIMI II).

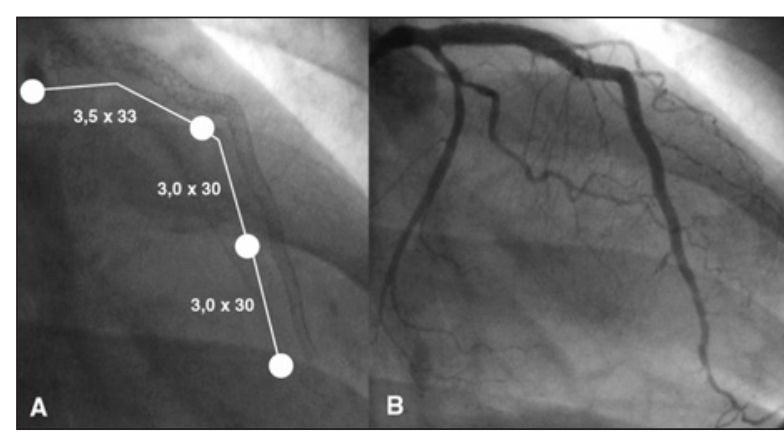

Figura 4 - Coronariografia após o implante de três stents com sobreposição de hastes na DA, guiado por USIC. Em A, grafia sem contraste mostrando a sombra da extensão dos stents. Em B, angiografia em OAD, mostrando resolução completa da dissecção e restauração do fluxo. 
aspirina $200 \mathrm{mg} /$ dia, ticlopidina $250 \mathrm{mg} 2 x /$ dia (por um mês), sinvastatina $40 \mathrm{mg} / \mathrm{dia}$ e atenolol $50 \mathrm{mg} / \mathrm{dia}$. Vem em acompanhamento clínico ambulatorial há quatro meses, permanecendo assintomática desde a alta hospitalar. Dosagens de marcadores inflamatórios e de distúrbios da coagulação (proteína C reativa, homocisteína, anticoagulante lúpico, anticorpos antinuclear e antimitocondrial), bem como dosagem de plaquetas, tempo de tromboplastina parcial e tempo de protrombina, foram realizadas no acompanhamento ambulatorial e se mostraram normais.

\section{DISCUSSÃO}

Condição mais freqüentemente encontrada em muIheres, a dissecção coronária espontânea apresenta-se numa proporção de 3 para 1 em relação aos homens, sendo $25 \%$ dos casos observados durante a gestação ou período pós-parto ${ }^{1-7}$.

No sexo feminino, as DCE ocorrem predominantemente na artéria coronária esquerda (87\%), geralmente acometendo mulheres jovens e sem fatores de risco coronariano. Contrariamente, os homens costumam ser acometidos numa faixa etária mais tardia e com presença de fatores de risco para DAC, envolvendo a CD em $67 \%$ dos casos. De maneira geral, a DA é afetada em $75 \%$ dos casos, a CD em 20\%, a Cx em $4 \%$ e o tronco da coronária esquerda (TCE) em menos de $1 \%$ dos $\operatorname{casos}^{2,5,8,9}$.

Os mecanismos etiopatogênicos exatos da dissecção coronária espontânea ainda não foram totalmente esclarecidos, parecendo ser multifatoriais.

Observa-se forte associação com a gestação, o puerpério e o uso de anticoncepcionais orais ${ }^{1-7,10}$.

Durante a gravidez, podem ocorrer alterações patológicas na parede arterial, em decorrência da fragmentação de fibras reticulares, hipertrofia de células musculares lisas e, alterações nos conteúdos de mucopolissacárides e composição de proteínas, levando ao enfraquecimento de sua parede e, por fim, sua ruptura durante o trabalho de parto, peri parto, ou pós-parto ${ }^{1,4}$.

Doenças imunológicas como lupus eritematoso sistêmico, doenças do colágeno (síndromes de Marfan e Ehler Danlos, principalmente, o tipo IV) e Doença de Kawasaki, podem estar relacionadas ${ }^{7}$.

São também relatadas associações com $\mathrm{HAS}^{11}$, exercício físico intenso ${ }^{12,13}$ e uso de cocaína ${ }^{14}$.

Um tipo especial de DCE é a que ocorre em vasos com ateromatose prévia. Estudos com USIC têm mostrado que a dissecção, nesses casos, resulta de ruptura espontânea da placa ateromatosa, por razões ainda desconhecidas. Acredita-se que pacientes com dissecções de etiologia ateromatosa tenham um prognóstico muito melhor, talvez em decorrência da presença de circulação colateral já desenvolvida ${ }^{7,15}$.
O plano onde a dissecção espontânea ocorre com mais freqüência é o terço externo da túnica média, ou entre a média e a adventícia ${ }^{3}$.

Achados histológicos em estudos post-mortem têm evidenciado presença de infiltrado inflamatório, principalmente eosinofílico, na túnica adventícia dos vasos em $25 \%$ a $40 \%$ dos pacientes que morreram por DCE. Proteases liberadas por eosinófilos podem promover reações proteolíticas nas paredes vasculares, podendo contribuir para dissecção ${ }^{7}$.

A necrose média da cística também pode ser vista em $25 \%$ a $30 \%$ dos casos estudados ${ }^{7}$, podendo estar presente na média da aorta ascendente de pacientes com Síndrome de Marfan, predispondo-os à dissecção aórtica' ${ }^{1}$.

Em alguns pacientes, tem-se identificado angiomatose da camada adventícia e, tem sido postulado que em alguns indivíduos o vasa vasorum das artérias coronárias são particularmente propensos à hemorragia espontânea, podendo ser o primeiro local de hemorragia na túnica média, resultando em hematoma intramural e dissecção ${ }^{1,7}$.

A forma de apresentação clínica pode englobar todo o espectro das síndromes coronarianas, dependendo da extensão da dissecção e do vaso envolvido. Morte súbita cardíaca é relatada em cerca de $50 \%$ dos casos, especialmente quando há dissecção do TCE ${ }^{3,5,7}$.

O prognóstico na fase precoce é usualmente ruim, dependendo de uma suspeita clínica e diagnóstico precoces e, quando viável, da realização de procedimentos de revascularização. Contudo, se a fase aguda é controlada, eventos subseqüentes são $\operatorname{raros}^{4}$.

O tratamento da DCE inclui a abordagem clínica, bem como modalidades invasivas, como a cirurgia de revascularização miocárdica (CRVM), ou angioplastia com implante de stents. Entretanto, devido à pequena quantidade de casos relatados na literatura, não existem estudos comparando as diversas formas de tratamento, sem existir consenso da utilização de uma forma ou outra de tratamento para determinado caso.

Drogas vasodilatadoras, como nitratos e bloqueadores dos canais de cálcio, diminuem a ocorrência de espasmo coronariano, prevenindo a extensão da dissecção, até a realização da revascularização do vaso ${ }^{7}$.

O papel dos fibrinolíticos ainda permanece controverso. Alguns trabalhos têm mostrado que pode ser benéfico, uma vez que pode dissolver trombos formados na falsa luz, que comprimiam a luz vascular com sua obliteração, restaurando o fluxo na luz verdadeira ${ }^{7}$. Entretanto, seu uso pode ser prejudicial em alguns casos, dificultando a cicatrização da dissecção, podendo, até mesmo, promover sua extensão. Buys et al. ${ }^{16}$ relataram o caso de uma mulher de 31 anos, admitida em síndrome coronariana aguda (SCA) causada por 
DCE, onde, após o uso de terapia trombolítica, houve agravamento da hemorragia na falsa luz e extensão da dissecção. Apesar desses relatos desfavoráveis, a maioria dos autores tem descrito bons resultados com a terapia clínica, incluindo os trombolíticos, em pacientes com quadro de IAM causados por DCE7.

A CRVM ainda é a intervenção terapêutica de escolha em dissecções que envolvem o TCE ou múltiplos vasos ${ }^{1,3,7,17}$. Contudo, a CRVM pode ser tecnicamente difícil, uma vez que a dissecção pode estar se estendendo mais distalmente do que evidenciado pela angiografia, dificultando o posicionamento do enxerto. Ademais, a dissecção pode se estender para os enxertos venosos e arteriais, bem como para as partes distais dos vasos revascularizados, comprometendo o resultado cirúrgico. Com isso, alguns autores recomendam que esta modalidade de tratamento somente seja utilizada em caso de falência do tratamento clínico agressivo ${ }^{1}$.

Alguns estudos têm mostrado sucesso com a angioplastia e implante de stents coronarianos, com resultados satisfatórios e baixa taxa de reestenose. Entretanto, este tipo de abordagem tem ficado reservado para casos com dissecção localizada e não muito extensa ${ }^{1,3}$, embora existam relatos de tratamento percutâneo com sucesso em DCE com acometimento de múltiplos va$\operatorname{sos}^{18}$. Uma das limitações da intervenção coronária percutânea pode ser a identificação da luz verdadeira ${ }^{3}$. Assim como para a CRVM, a extensão muito distal da dissecção, em locais onde o diâmetro do vaso acometido seja menor que $2 \mathrm{~mm}$, também pode configurar limitação ao implante de stents.

O caso apresentado merece algumas considerações. Trata-se de uma mulher jovem, com antecedentes de HAS e tabagismo, admitida em SCA SSST com dissecção espontânea da DA, que evoluiu desfavoravelmente durante o seguimento clínico, com necessidade de revascularização do vaso em caráter de urgência.

Contrariando os dados da literatura, não havia associação com gravidez, puerpério ou uso de anticoncepcionais orais. Apesar de portar alguns fatores de risco para DAC, o estudo angiográfico não demonstrou presença de ateromatose coronária significativa.

O quadro inicial de SCA pode ter sido causado pelo referido espasmo, ou por uma dissecção incipiente na DA, não visível na angiografia inicial. A administração de medicações antiagregantes e antitrombóticas, como parte do tratamento de SCA SSST, pode ter favorecido a formação de hematoma na falsa luz, agravando e estendendo a dissecção.

Diversos relatos têm demonstrado a presença de infiltrados inflamatórios e hipertrofia de células musculares lisas na parede dos vasos acometidos. Essas alterações, além de poderem estar envolvidas na fisiopatologia da DCE, poderiam, teoricamente, contribuir para o desenvolvimento de espasmo coronário. Contudo, não encontramos na literatura relatos sobre a associação destas duas condições.

Apesar de parecer recomendação geral de que a angioplastia com implante de stents seja reservada para os casos de acometimento uniarterial, ou com dissecções localizadas, nosso caso mostrou uma dissecção extensa desde o óstio da DA até seu terço distal. A identificação da luz verdadeira foi possível e sem dificuldades. O uso do USIC durante o procedimento foi útil em mostrar a verdadeira extensão da dissecção, além de otimizar o implante das próteses. O implante de três stents longos e com sobreposição de hastes foi feito sem complicações. Até o seguimento de quatro meses, a paciente encontra-se assintomática, sem sinais clínicos que sugiram a possibilidade de reestenose coronária, fato que poderia ser preocupante pela extensão do segmento de stents não farmacológicos. Não encontramos na literatura relato de tratamento de DCE extensa pela via percutânea.

\section{REFERÊNCIAS BIBLIOGRÁFICAS}

1. Maresta A, Varani E, Balducelli M, Vecchi G. Spontaneous coronary dissection of all three coronary arteries: a case description with medium-term angiographic follow-up. Ital Heart J 2002;3:747-51.

2. Narasimhan S. Spontaneous coronary artery dissection (SCAD). IJTCVS 2004;20:189-91.

3. Kar S, Shah PK. Acute coronary syndrome caused by coronary artery dissection mimicking acute plaque rupture. Rev Cardiovasc Med 2001;2:215-9.

4. De Maio SJ Jr, Kinsella SH, Silverman ME. Clinical course and long-term prognosis of spontaneous coronary artery dissection. Am J Cardiol 1989;64:471-4.

5. Basso C, Morgagni GL, Thiene G. Spontaneous coronary artery dissection: a neglected cause of acute myocardial ischaemia and sudden death. Heart 1996;75:451-4.

6. Almeda FQ, Barkatullah S, Kavinsky CJ. Spontaneous coronary artery dissection. Clin Cardiol 2004;27:377-80.

7. Hamilos MI, Kochiadakis GE, Skalidis El, Igoumenidis NE, Chrysostomakis SI, Vardakis KE, et al. Acute myocardial infarction in a patient with spontaneous coronary artery dissection. Hellenic J Cardiol 2003;44:348-51.

8. Jorgensen MB, Aharonian V, Mansukhani P, Mahrer PR. Spontaneous coronary dissection: a cluster of cases with this rare finding. Am Heart J 1994;127:1382-7.

9. Virmani R, Forman MB, Robinowitz M, McAllister HA Jr. Coronary artery dissections. Cardiol Clin 1984;4:633-46.

10. Azam MN, Roberts DH, Logan WF. Spontaneous coronary artery dissection associated with oral contraceptive use. Int J Cardiol 1995;48:195-8.

11. Greenblatt JM, Kochar GS, Albornoz MA. Multivessel spontaneous coronary artery dissection in a patient with severe systolic hypertension: a possible association. A case report. Angiology 1999;50:509-13.

12. Sherrid MV, Mieres J, Mogtader A, Menezes N, Steinberg G. Onset during exercise of spontaneous coronary artery dissection and sudden death. Occurrence in a trained athlete: case report and review of prior cases. Chest 1995;108:284-7. 
Ishii EY, et al. Angioplastia com Implante de Stents para o Tratamento de Dissecção Coronária Espontânea. Rev Bras Cardiol Invas 2007; 15(1): 77-81.

13. Ellis CY, Haywood GA, Monro JL. Spontaneous coronary artery dissection in a young woman resulting from an intense gymnasium "work out". Int J Cardiol 1994;47:193-4.

14. Jaffe BD, Broderick TM, Leier CV. Cocaine-induced coronary artery dissection. N Engl J Med 1994;330:510-1.

15. Maehara A, Mintz GS, Castagna MT, Pichard AD, Satler LF, Waksman R, et al. Intravascular ultrasound assessment of spontaneous coronary artery dissection. Am J Cardiol 2002; 89:466-8.
16. Buys EM, Suttorp MJ, Morshuis WJ, Plokken HW. Extension of a spontaneous coronary artery dissection due to thrombolytic therapy. Cathet Cardiovasc Diagn 1994;33:157-60.

17. Vicari R, Eybel C, Monson D. Survival following spontaneous coronary artery dissection: surgical repair by extrusion of intramural hematoma. Am Heart J 1986;111:593-4.

18. Togni M, Amann FW, Follath F. Spontaneous multivessel coronary artery dissection in a pregnant woman treated successfully with stent implantation. Am J Med 1999;107:407-8 